\title{
Paracoccidioidomicose juvenil: apresentação atípica e disseminada em adulto do sexo feminino.
}

\author{
Juvenile paracoccidioidomycosis: atypical and disseminated \\ presentation in female adult.
}

1 Júlia Pires dos Reis Maia ju.prmaia@gmail.com

1 Marina Silva Guedes

1 Thamires Aparecida Pereira Noronha

1 Luiz Henrique Conde Sangenis

1 Faculdade de Medicina de Valença

\section{Resumo}

Paracoccidioidomicose é uma doença micótica sistêmica causada por um fungo termodimórfico do gênero Paracoccidioides, também conhecida no passado como Micose de Lutz ou Blastomicose Sul-Americana. Com maior ocorrência nas regiões Sul, Sudeste e Centro-oeste principalmente em zona rural e em locais de desmatamento recente. 0 caso reportado ocorreu no município de Valença-RJ e contraria o perfil epidemiológico e de manifestação clínica mais comum da doença. Neste relato discutimos a importância do diagnóstico diferencial, a instituição do tratamento correto e analisamos a diferença de ocorrência da doença entre os sexos.

\section{Palavras-chaves}

Paracoccidioidomicose. Mulher. Clínica. Epidemiologia. Imunologia.

\begin{abstract}
Paracoccidioidomycosis is a systemic fungal disease caused by a thermodymorphic fungus of the genus Paracoccidioides, also known in the past as Lutz mycosis or South American blastomycosis. Most occurring in the South, Southeast and Center-West regions, mainly in rural areas and in recent deforestation sites. The case reported here occurred in the city of Valença-RJ and contradicts the epidemiological profile and the most common clinical manifestation of the disease. In this report we discussed the importance of the differential diagnosis and the implementation of the correct treatment and analyzed the difference in the occurrence of the disease between the sexes.
\end{abstract}

\section{Keywords}

Paracoccidioidomycosis. Woman. Clinical. Epidemiology. Immunologogy

\section{Como você deve citar?}

MAIA, Júlia Pires dos Reis et al. Paracoccidioidomicose juvenil: apresentação atípica e disseminada em adulto do sexo feminino. Cadernos UniFOA, Volta Redonda, n. 41, p. 127-136, dezembro 2019. 


\section{INTRODUÇÃO}

A Paracoccidioidomicose (PCM) é uma doença micótica sistêmica causada por um fungo termodimórfico do gênero Paracoccidioides, sendo duas espécies reconhecidas: Paracoccidioides brasiliensis e Paracoccidioides lutzii (NUCCl; COLOMBO, 2018).

O fungo tem uma distribuição geográfica limitada a América Central e do Sul, onde a PCM é endêmica e a micose sistêmica mais frequente. Ocorrendo principalmente no Brasil, Colômbia, Venezuela e Argentina.

Paracoccidioides brasiliensis vive, saprofiticamente em solos férteis, úmidos e com abundante vegetação (CAPONE et al., 2010). O principal fator de risco para adquirir a infecção é ter profissão ou atividade relacionada ao manejo do solo contaminado com fungos (SHIKANAI-YASUDA et al., 2017).

Na infância, a PCM é uniformemente distribuída entre ambos os sexos, com uma suave predominância em indivíduos do sexo masculino; contudo, entre adolescentes e na vida adulta, a frequência varia de 10 a 15 homens para uma mulher (SHIKANAI-YASUDA et al., 2017). Esta diferença na incidência entre homens e mulheres pode ser explicada, pela proteção conferida pelos hormônios sexuais femininos (BELLISSIMO-RODRIGUES et al., 2013; SEVERO et al., 1998; SHANKAR et al., 2011) e devido à realização de trabalhos rurais serem executados principalmente por homens (BELLISSIMO-RODRIGUES et al., 2013).

A PCM pode se apresentar na forma aguda/subaguda (juvenil), forma crônica (adulto) e em alguns casos, apresentar manifestações de ambas as formas, sendo considerada mista (SHIKANAI-YASUDA et al., 2017). A infecção por Paracoccidioides brasiliensis afeta primariamente os pulmões, através da inalação de conídios do fungo e pode espalhar-se para vários órgãos e sistemas, originando mais comumente lesões em mucosas, linfonodos, pele e glândulas adrenais. Os indivíduos afetados podem apresentar sintomas gerais, incluindo febre, perda ponderal, astenia e prostração. 0 trato digestivo não é usualmente afetado, correspondendo a aproximadamente $2,7 \%$ dos casos de PCM (GALEAZZI et al., 2011).

O objetivo deste estudo foi descrever a ocorrência da PCM na forma aguda disseminada, incluindo acometimento pulmonar em adulto do sexo feminino internada no setor de Clínica Médica do Hospital Escola Luiz Gioseffi Jannuzzi em Valença - RJ no período de 26/09/2017 a 10/11/2017 e através da revisão de literatura, discutir aspectos da doença no sexo feminino em comparação ao sexo masculino.

\section{ASPECTOS ÉTICOS}

Este trabalho foi submetido ao Comitê de Ética em Pesquisa (CEP) da Faculdade de Medicina de Valença-RJ e foi aprovado com número de Certificação de Apresentação para Apreciação Ética (CAAE) 01688418.0.0000.5246 e número de parecer de aprovação 3.003.079.

\section{RELATO DE CASO}

JS, sexo feminino, 36 anos, negra, técnica de enfermagem, natural e residente em Valença-RJ, onde reside na área periurbana. Em setembro de 2017 foi admitida no Hospital Escola Luiz Gioseffi Jannuzzi (HELGJ) com relato de tosse por três semanas, inicialmente seca e posteriormente produtiva, com expectoração clara, febre de $38,8^{\circ}$ centígrados, dispneia aos moderados esforços, linfonodomegalia cervical e perda ponderal não mensurada nos últimos três meses. Portadora de hipertensão arterial sistêmica e síndrome do pânico. Etilista social e tabagista (CT 3 anos maços). A hipótese diagnóstica inicial foi tuberculose, sendo iniciado tratamento com RIPE (rifampicina, isoniazida, pirazinamida, etambutol). 
No exame físico admissional, a paciente apresentava-se hipocorada, febril, taquipneica, taquicárdica e com dessaturação de oxigênio em ar ambiente. A ausculta pulmonar era universalmente diminuída com roncos difusamente distribuídos em ambos os pulmões. Apresentava linfonodomegalia cervical bilateral, móvel, semi endurecida e sem fistulização.

Exames laboratoriais admissionais demonstravam: hemácias 4,64 milhões $/ \mathrm{mm}^{3}$, hemoglobina $10,7 \mathrm{~g} \%$, hematócrito 32,1\%, HCM 23pg, VCM 70,47 U $, \mathrm{CHCM} 32,72 \%$, Leucograma 6.700/mm³ (0-3/0-0-3-57/34-3), plaquetas $569 \mathrm{mil} / \mathrm{mm}^{3}$, uréia $17 \mathrm{mg} / \mathrm{dl}$, creatinina $0,7 \mathrm{mg} / \mathrm{dl}$, sódio $127 \mathrm{mmol} / \mathrm{L}$ e potássio $3,9 \mathrm{mmol} / \mathrm{L}$. Radiografia de tórax em PA revelou padrão micronodular difuso em ambos os pulmões (Figura 1)

A pesquisa de bacilos-álcool-ácido-resistentes (BAAR) em três amostras de escarro foi negativa. Testes sorológicos para pesquisa de marcadores de hepatites $\mathrm{B}$ e $\mathrm{C}$ e anti-HIV resultaram negativos e a tomografia computadorizada do tórax revelou a presença de incontáveis micronódulos distribuídos difusamente por ambos os pulmões, de aspecto não específico, compatível com processo infeccioso, tuberculose ou histoplasmose, ou metástase hematogênica. Aumento dos linfonodos peri-hilares.

Diante da possibilidade de neoplasia, foi realizada tomografia computadorizada do abdômen para rastreamento, mesmo sem quaisquer sintomas intestinais, revelando moderado espessamento da parede do ceco, cólon ascendente e parte do cólon transverso, com discreto aumento da densidade da gordura adjacente, reduzindo sua luz, porém sem determinar obstrução intestinal, de aspecto pouco específico, mas que poderia corresponder a lesão de origem neoplásica inflamatória, fortalecendo a hipótese de neoplasia. Ultrassonografia total de abdômen também foi realizada sem demonstrar alterações.

Dando continuidade à investigação, foi realizada colonoscopia para esclarecimento dos achados tomográficos que evidenciou: ileíte distal associada a pancolíte, sendo interrogada causa inflamatória e tuberculose intestinal (Figura 2). Fazendo com que a possibilidade de tuberculose pulmonar, e agora intestinal, se tornasse mais contundente.

Realizada também biópsia ganglionar cervical para estudo histopatológico, cujo resultado demorou 20 dias. Neste período, a paciente não apresentou melhora clínica, mantendo episódio febril vespertino ou durante período noturno.

Como não foi observada resposta ao tratamento contra a tuberculose, foi solicitada contra-imunoeletroforese para pesquisa de criptococose, histoplasmose e PCM. Também foram realizados: raspado de mucosa bucal e coleta de escarro para pesquisa de fungo, além de nova pesquisa de BAAR, o que resultaram negativos.

A paciente apresentou lesões sugestivas de candidíase oral, sendo tratada com nistatina tópica. No vigésimo terceiro dia de internação hospitalar foi iniciada anfotericina B empiricamente para PCM, o que acabou se confirmando dois dias após com o resultado histopatológico do material colhido através da biópsia ganglionar, demonstrando reação inflamatória granulomatosa contendo vários exemplares de Paracoccidioides brasiliensis.

Após cinco dias do início da anfotericina B, a paciente apresentou hipotensão arterial persitente, sendo então considerada hipótese de insuficiência adrenal, sendo iniciada corticoterapia endovenosa. Após 15 dias de uso da anfotericina B, com dose acumulada de $750 \mathrm{mg}$, a paciente apresentou queda importante de hemoglobina e hematócrito, 6,4 e 20 respectivamente, por provável toxidade hematológica ao fármaco. Optou-se pela troca da anfotericina B por itraconazol na dose de $200 \mathrm{mg} / \mathrm{dia}$, apresentando boa resposta clínica: sem episódios febris e melhora do estado geral. Neste período, a contra-imunoeletroforese resultou positiva para PCM e negativa para histoplasmose e criptococose. 
Na ocasião da alta hospitalar, no quadragésimo sexto dia de internação, o esquema terapêutico foi substituído por sulfametoxazol-trimetoprim, devido à disponibilidade no SUS e mantido seguimento ambulatorial, onde demonstrou ganho ponderal e melhora significativa do estado geral. No acompanhamento ambulatorial apresentou remissão da tosse, melhora da ausculta pulmonar, normalização radiológica e dos exames laboratoriais. Atualmente encontra-se no segundo ano de tratamento.

Figura 1 - Comparativos entre radiografias de tórax em PA na entrada em setembro de 2017 com micronódulos difusos no parênquima e após quatro meses de tratamento antifúngico em fevereiro de 2018 com transparência normal.

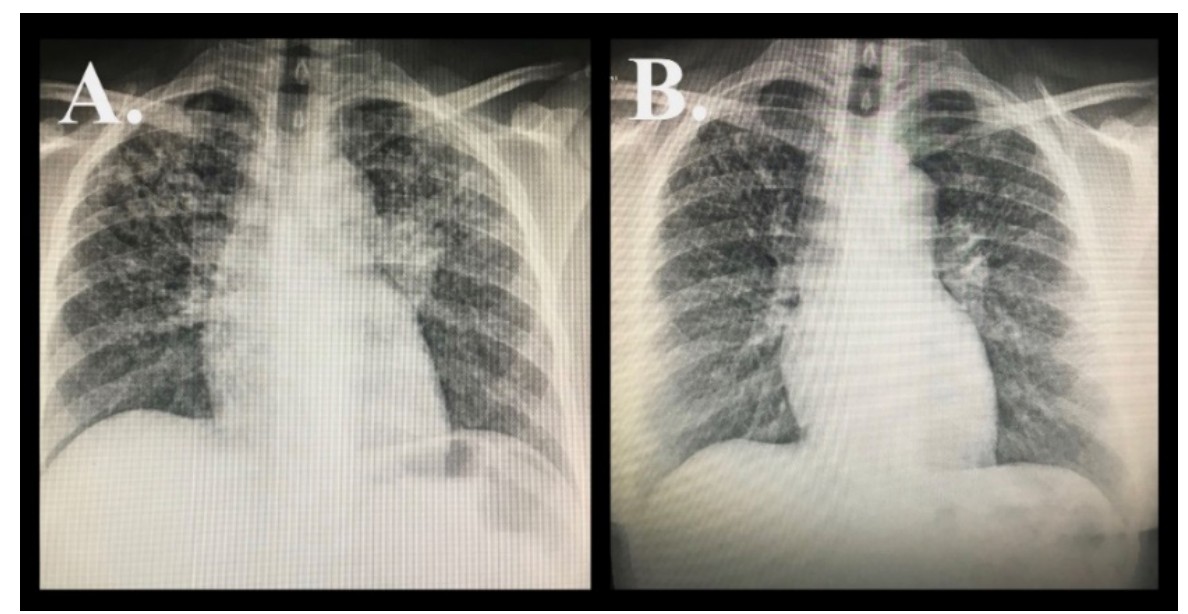

Fonte: Dos autores, 2019

Figura 2 - Imagens da colonoscopia realizada na paciente JS demonstrando lesões sugestivas de ileíte e pancolíte.

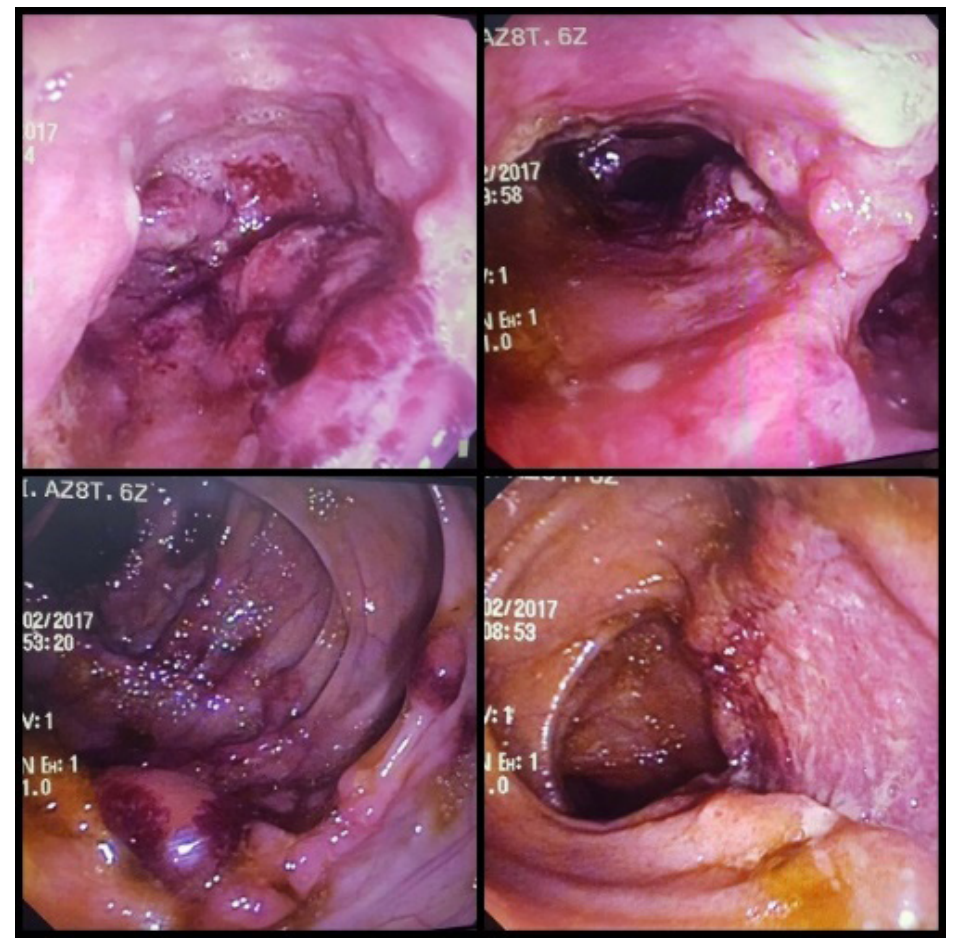

Fonte: Dos autores, 2019. 


\section{DISCUSSÁO}

A PCM assim como outras doenças fúngicas sistêmicas, não é de notificação compulsória (MINISTÉRIO DA SAÚDE, 2009), o que reduz os registros da doença e dificulta saber com exatidão sobre dados de prevalência e incidência, sendo estas subestimadas (VALLE et al., 2017).

Baseado em relatos de casos clínicos e outros registros nota-se uma maior ocorrência da doença nas regiões Sul, Sudeste e Centro-oeste, sendo de caráter endêmico em populações de zona rural. Novos registros nos estados do Amazonas, Maranhão, Tocantins, Pará, Mato Grosso, Rondônia e Acre têm ocorrido e têm associação com o desmatamento (MINISTÉRIO DA SAÚDE, 2009). A cidade de Valença está localizada na região do vale do rio Paraíba do Sul no estado do Rio de Janeiro, onde há registros de outros casos.

A região do Vale do Rio Paraíba do Sul é formada por uma planície nas bordas do rio, entre a Serra da Mantiqueira e a Serra do Mar, onde a umidade é de aproximadamente $75 \%$. A conjunção de área de floresta com água abundante é a condição ideal para o crescimento de Paracoccidioides spp. Inicialmente a área de mata, foi parcialmente substituída por plantação de café, seguida de pastagens e pequena áreas de plantações de cana de açúcar, vegetais e frutas (RIOS GONÇALVES et al., 1998).

Recentemente, foram observados no estado do Rio de Janeiro, oito casos da forma aguda/subaguda de PCM após a construção da rodovia Raphael de Almeida Magalhães (Arco Metropolitano) entre 2008 e 2014, quando houve desflorestamento, remoção intensa de terra e período abundante de chuvas. Nesse estudo, a razão de incidência entre o sexo masculino:feminino foi de 1:1 e a faixa etária variável entre 10 e 28 anos, tendo como tempo médio de diagnóstico sete meses (VALLE et al., 2017). Assim como no caso tema deste relato, todos os pacientes apresentaram linfonodomegalias. Os autores acreditam que outras pessoas também expostas a Paracoccidioides spp. poderão desenvolver a forma crônica da doença futuramente (VALLE et al., 2017).

A infecção, geralmente, é adquirida nas duas primeiras décadas de vida, com pico de incidência entre 10 e 20 anos de idade. As manifestações clínicas são comuns em adultos entre 30 e 50 anos de idade, incidindo mais em homens do que em mulheres (SHIKANAI-YASUDA et al., 2017).

Neste caso observamos a ocorrência da doença contrariando diversos aspectos epidemiológicos, tendo ocorrido em paciente do sexo feminino, moradora de área urbana periférica, sem contato com atividades agrícolas e mesmo em seu passado não havia relato de contato com zona rural, nem mesmo sua profissão permite estabelecer relação com a doença, visto que a mesma é profissional da área da saúde, atuando como técnica de enfermagem.

A apresentação da doença foi classificada como forma aguda e na ocasião da internação, a paciente tinha aproximadamente três semanas de sintomas gerais e respiratórios além de linfonodomegalias cervicais, tornando plausível a hipótese de tuberculose.

Pacientes que desenvolvem a forma pulmonar da doença podem apresentar sintomas e sinais respiratórios, bem como sintomas inespecíficos. Como nem todos os hospitais dispõem de exames microbiológicos em suas rotinas, muitos pacientes acabam sendo tratados empiricamente para tuberculose. Exames diagnósticos específicos como a imunodifusão dupla para fungos e exames micológicos diretos, devem ser rotineiramente utilizados nas infecções respiratórias que não respondem a terapia específica para tuberculose (FERREIRA-DA-CRUZ; WANKE; GALVÃO-CASTRO, 1987).

Neste caso, a paciente não apresentou melhora com tratamento empírico e não confirmou diagnóstico de tuberculose nos exames específicos, tornando a hipótese de PCM mais evidente. Muito embora possa haver 
associação das duas doenças em cerca de 10\% dos pacientes acometidos (SHIKANAI-YASUDA et al., 2017). De acordo com o tempo de evolução descrito e os sinais clínicos observados, o caso era compatível com a forma aguda/subaguda da PCM, menos frequente (5-25\%), comparado com a forma crônica, com predomínio de casos na infância, sendo raro em adultos (SHIKANAI-YASUDA et al., 2017).

Lacaz et al. (1982), citado por RIOS GONÇALVES et al. (2000), demonstraram três casos de PCM na faixa etária entre 1 e 7 anos, para cada 1.000 adultos e jovens de mais de 14 anos com a doença. Crianças têm suas atividades restritas ao redor de suas casas, indicando que o fungo Paracoccidioides spp. provavelmente vive em áreas próximas de residências rurais na região do Vale do Paraíba Fluminense (RIOS GONÇALVES et al., 1998).

Terra (1993), citado por RIOS GONÇALVES et al. (2000), reviu 30 casos infantis, do estado do Rio de Janeiro, sendo a maioria proveniente do Vale do Paraíba Fluminense, considerando pequeno o deslocamento migratório de crianças, esse aspecto é importante para delinear zonas endêmicas.

As manifestações respiratórias foram os principais sintomas nesta paciente, sugerindo acometimento pulmonar, o que também é raro na forma aguda de apresentação da doença. Esta se apresenta principalmente com comprometimento disseminado de órgãos do sistema linforreticular, com presença de adenomegalia e hepatoesplenomegalia, podendo também ocorrer manifestações osteoarticulares, cutâneas e digestivas (FERREIRA et al., 2013).

A paciente em questão não apresentava sintomas e sinais clínicos gastrointestinais, sendo este um achado nos exames de imagem e diagnóstico de exclusão, visto que inicialmente a tomografia de abdômen sugeria possível neoplasia e as imagens da colonoscopia tuberculose intestinal, demonstrando a importância desses exames complementares. A tomografia computadorizada tem aumentado as chances de diagnosticar casos de paracoccidioidomicose, inclusive intestinal, demonstrando alterações antes descritas somente por radiografia convencional, laparotomia ou necropsia (CHOJNIAK et al. 2000). O diagnóstico intestinal foi confirmado apenas com a biópsia e anteriormente a paciente já havia sido diagnosticada com PCM pela biópsia ganglionar.

Estudos de série de casos de Barbosa et al. (1968) citados por RIOS GONÇALVES et al. (2000) demonstraram ser comum o comprometimento intestinal na PCM até a terceira década de vida e em indivíduos do sexo masculino, porém não foi constado lesão pulmonar nas radiografias de tórax, o que diferiu do caso aqui relatado. Já Andrade (1983), também citado por RIOS GONÇALVES et al. (2000), constatou acometimento linfático abdominal no intestino, baço e fígado, o que não se demonstrou em exames de imagem no presente relato, estando restrito à mucosa intestinal.

Neste caso, há ocorrência da PCM no sexo feminino, em idade fértil, cujos níveis de estrogênio deveriam estar atuando como fator protetor. Após revisão dos últimos 10 anos, nas plataformas PubMed, MEDLINE - Bireme, SciELO e LILACS, todos os estudos analisados certificaram estatisticamente a maior incidência da doença no sexo masculino, exceto um que demonstrou equivalência na razão de incidência entre os sexos (Tabela 1). 
Tabela 1 - Comparativo entre os estudos de série de casos de PCM revisados no período de 2008 a 2017 e suas razões de incidência entre os sexos masculino/feminino.

\begin{tabular}{|c|c|c|c|c|c|}
\hline Autores e data & Local & $\begin{array}{l}\mathrm{N}^{\circ} \text { total de } \\
\text { pacientes }\end{array}$ & Masculino & Feminino & $\begin{array}{c}\text { Razão masculino: } \\
\text { feminino }\end{array}$ \\
\hline $\begin{array}{l}\text { Belíssimo-Rodrigues, } \\
\text { Machado e Martinez } \\
\text { (2011) }\end{array}$ & Sudeste (BR) & 1000 & 855 & 145 & $5,8: 1$ \\
\hline $\begin{array}{l}\text { Belíssimo-Rodrigues et } \\
\text { al. (2013), }\end{array}$ & Sudeste (BR) & 1219 & 1048 & 171 & $6,12: 1$ \\
\hline Fabris et al. (2014) & $\begin{array}{l}\text { Centro-Oeste } \\
\text { (BR) }\end{array}$ & 595 & 549 & 46 & 11,9:1 \\
\hline Loth et al. (2011) & Sul (BR) & 102 & 72 & 30 & $2,4: 1$ \\
\hline Macedo et al. (2017) & Sudeste (BR) & 29 & 14 & 15 & $1: 1,07$ \\
\hline Matos et al. (2012) & Nordeste (BR) & 245 & 219 & 26 & $8,4: 1$ \\
\hline Sangenis (2008) & Sudeste (BR) & 78 & 72 & 6 & $12: 1$ \\
\hline Shankar et al. (2011) & $\begin{array}{l}\text { Brasil e } \\
\text { Colômbia }\end{array}$ & 5.562 & 5.105 & 457 & $11,1: 1$ \\
\hline $\begin{array}{c}\text { Souza, Jorge e } \\
\text { Orzechowski Xavier } \\
(2014)\end{array}$ & Sul (BR) & 123 & 104 & 19 & $5,4: 1$ \\
\hline Viera et al. (2014) & Norte (BR) & 2.163 & 1952 & 211 & $9,2: 1$ \\
\hline
\end{tabular}

Fonte: Dos autores, 2019.

\section{CONCLUSÃO}

A PCM é uma doença sistêmica com amplo espectro de apresentação clínica e de diagnóstico diferencial. $O$ presente estudo traz relevância ao demonstrar caso de PCM com apresentação atípica, mostrando a necessidade de sempre considerar o diagnóstico diferencial, principalmente com quadros sugestivos de tuberculose pulmonar, intestinal ou disseminada, mesmo em pacientes que não residam em áreas rurais ou de colonização recente, que não tenham atividade laboral característica ou que sejam do sexo feminino.

Os estudos analisados na revisão de literatura dos últimos 10 anos certificam estatisticamente a maior incidência da doença no sexo masculino, demonstrando a peculiaridade do caso aqui reportado: paciente adulta do sexo feminino com evolução aguda, deterioração rápida do estado geral e disseminação para vários sistemas orgânicos. 
A PCM tem alto potencial para morbidade, mortalidade e sequelas, sendo fundamental o diagnóstico precoce, o estadiamento clínico-laboratorial e os exames de imagens para melhor investigação de complicações. A abordagem terapêutica deve ser individualizada de acordo com a apresentação clínica, o padrão de tolerabilidade e adesão de cada paciente. Cabe ainda considerar a observação de mudanças epidemiológicas, com a ocorrência da doença fora de áreas rurais e em indivíduos do sexo feminino.

\section{AGRADECIMENTOS}

Ao staff médico, Dr. Leonardo Rebouças Novaes, Dr. Ricardo Rocco, Dra. Ivy Menezes Monteiro, Dr. Thompson Baptista Machado Junior, Dra. Renata Valente Lisboa e demais profissionais do setor de Clínica Médica do HELGJ pela ajuda incondicional durante a assistência ao caso e incentivo para elaboração deste artigo.

\section{REFERÊNCIAS}

BELLISSIMO-RODRIGUES, F.; MACHADO, A. A.; MARTINEZ, R. Paracoccidioidomycosis Epidemiological Features of a 1,000-Cases Series from a Hyperendemic Area on the Southeast of Brazil. Am. J. Trop. Med. Hyg. Ribeirão Preto, v. 85, n. 3, set. 2011. Disponível em: <https://www.ncbi.nlm.nih.gov/pmc/ articles/PMC3163882/>. Acesso em: 02 out. 2018.

BELLISSIMO-RODRIGUES, F. et al. Endemic paracoccidioidomycosis: relationship between clinical presentation and patients' demographic features. Medical Mycology. Ribeirão Preto, v. 51, n. 3, abr. 2013. Disponível em: <https://academic.oup.com/mmy/article/51/3/313/1049516>. Acesso em: 01 abr. 2018.

CAPONE, D. et al. Micoses Pulmonares. Revista Hospital Universitário Pedro Ernesto. Rio de Janeiro, v. 9, n. 2, dez. 2010. Disponível em: <http://revista.hupe.uerj.br/detalhe_artigo.asp?id=163>. Acesso em: 19 ago. 2018.

CHOJNIAK, R. et al. Intestinal Paracoccidioidomycosis Simulating Colon Cancer. Rev. Soc. Bras. Med. Trop. Uberaba, v. 33, n. 3, jun. 2000. Disponível em: <http://www.scielo.br/scielo.php?script=sci_ arttext\&pid=S0037- 86822000000300010\&lng=en\&nrm=iso>. Acesso em: 07 jun. 2018.

FABRIS, L. R. et al. Decreasing Prevalence of the Acute/Subacute Clinical Form of Paracoccidioidomycosis in Mato Grosso do Sul State, Brazil. Rev Inst Med Trop Sao Paulo. São Paulo. v. 56, n.2, mar-abr. 2014. Disponível em: <https://www.ncbi.nlm.nih.gov/pmc/articles/PMC4085850/〉. Acesso em: 02 out. 2018.

FERREIRA, C. M. S. D. et al. Paracoccidioidomicose Subaguda Abdominal em Paciente Adulto Imunocompetente. Relato de caso. Rev Bras Clin Med. São Paulo, v. 11, n. 2, jun. 2013. Disponível em: <http://files.bvs.br/upload/S/1679-1010/2013/v11n2/a3577.pdf . Acesso em: 07 jun. 2018.

FERREIRA-DA-CRUZ, M.F.; WANKE, B.; GALVÃO-CASTRO, B. Prevalence of Paracoccidioidomycoses in Hospitalizes Adults in Rio de Janeiro (RJ), Brazil. Mycopathologia. Dordrecht. v. 97, n. 1, 1987. Disponível em: <https://www.ncbi.nlm.nih.gov/pubmed/3104788>. Acesso em: 27 jan. 2019

GALEAZZI, C. J. et al. Fungal Colitis by Paracoccidioides brasiliensis: a case report. J. Coloproctol. (Rio J.), Rio de Janeiro, v. 31, n. 4, dez. 2011. Disponível em: <http://www.scielo.br/scielo.php?script=sci_ arttext\&pid=S2237-93632011000400013\&lng=en\&nrm=iso>. Acesso em: 24 jul. 2018. 
LOTH, E. A. et al. Occurrence of 102 Cases of Paracoccidioidomycosis in 18 months in the Itaipu Lake Region, Western Paraná. Rev Soc Bras Med Trop, Uberaba, v. 44, n. 5, set-out. 2011. Disponível em: $<$ http://www.scielo.br/scielo.php?script=sci_arttext\&pid=S0037-86822011000500023>. Acesso em: 01 out. 2018.

MACEDO, P. M. et al. Acute juvenile Paracoccidioidomycosis: A 9year cohort study in the endemic área of Rio de Janeiro, Brazil. PLoS Negl Trop Dis, San Diego, v. 11, n. 3, mar. 2017. Disponível em: <https:// www.ncbi.nlm.nih.gov/pmc/articles/PMC5386294/pdf/pntd.0005500.pdf>. Acesso em: 01 out. 2018.

MATOS, W. B. et al. Paracoccidioidomycosis in the State of Maranhão, Brazil: Geographical and Clinical Aspects. Rev Soc Bras Med Trop, Uberaba, v. 45, n. 3, maio-jun. 2012. Disponível em: <http://www. scielo.br/scielo.php?script=sci_arttext\&pid=S0037-86822012000300020 . Acesso em: 01 out. 2018.

MINISTÉRIO DA SAÚDE. Guia de Vigilância Epidemiológica. Brasília. 2009. Disponível em: <https://www. infectologia.org.br/admin/zcloud/principal/2016/06/guia_vigilancia_epidemio_2010.pdf z. Acesso em: 24 jan. 2019

NUCCI, M.; COLOMBO, A. L. Clinical Manifestations and Diagnosis of Acute/Subacute Paracoccidioidomycosis. UpToDate. mar. 2018. Disponível em: <https://www.uptodate.com/contents/clinical-manifestationsand-diagnosis-of-acute-subacute-paracoccidioidomycosis?source=history_widget $>$. Acesso em: 28 jul. 2018.

RIOS GONÇALVES, A. J. et al. Paracoccidioidomycosis in Children in the State of Rio de Janeiro (Brazil). Geographic Distribution and the Study of "Reservarea". Rev. Inst. Med. Trop. S. Paulo. São Paulo. v. 40, n. 1. jan-fev. 1998. Disponível em: <http://www.scielo.br/scielo.php?script=sci arttext\&pid=S0036-46651998000100003>. Acesso em: 27 jan. 2019.

RIOS GONÇALVES, A. J. et al. Paracoccidioidomicose Infanto-Juvenil. Relato de Dez Pacientes Recentemente Observados na Cidade do Rio de Janeiro. Revisão da Literatura. Rev Med HSE. Rio de Janeiro. v. 1, n.34. 2000. Disponível em: <http://www.hse.rj.saude.gov.br/profissional/revista/34/parac. asp>. Acesso em: 28 jan. 2019.

SANGENIS, L. H. C. Mesa Redonda: Micoses Sistêmicas, Paracoccidioidomicose. II Congresso de Infectologia da Sociedade de Infectologia do Estado do Rio de Janeiro. 2008. Rio de Janeiro.

SEVERO, L. C. et al. Paracoccidioidomycosis in Women. Rev Iberoam Micol. Porto Alegre. v. 15, n. 2, jun. 1998. Disponível em: <https://www.ncbi.nlm.nih.gov/pubmed/17655417>. Acesso em: 01 out. 2018.

SHANKAR, J. et al. Hormones and the Resistance of Women to Paracoccidioidomycosis. Clin Microbiol Rev. San Jose. v. 24, n. 2, abr. 2011. Disponível em: <https://www.ncbi.nlm.nih.gov/pmc/articles/ PMC3122492/>. Acesso em: 01 out. 2018.

SHIKANAI-YASUDA, M. A. et al. Brazilian guidelines for the clinical management of paracoccidioidomycosis. Rev. Soc. Bras. Med. Trop., Uberaba, v. 50, n. 5, set. 2017. Disponível em: $<$ http://www.scielo.br/scielo.php?script=sci_arttext\&pid=S0037-86822017000500715\&lng=en\&nrm= iso>. Acesso em: 28 jul. 2018.

SOUZA, S. P.; JORGE, V. M.; ORZECHOWSKI XAVIER, M. Paracoccidioidomycosis in Southern Rio Grande do Sul: A Retrospective Study of Histopathologically Diagnosed Cases. Braz. J. Microbiol. São Paulo. 
v. 45, n. 1, 2014. Disponível em: <http://www.scielo.br/scielo.php?script=sci_isoref\&pid=S1517-

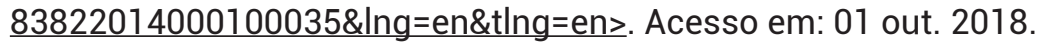

VALLE, A. C. F. et al. Paracoccidioidomycosis After Highway Construction, Rio de Janeiro, Brazil. Emerging Infectious Diseases. Atlanta. v. 23, n. 11, nov. 2017. Disponível em: <https://wwwnc.cdc.gov/ eid/article/23/11/17-0934_article>. Acesso em: 01 fev. 2019.

VIEIRA, G. D. et al. Paracoccidioidomycosis in a western Brazilian Amazon State: Clinical-epidemiologic Profile and Spatial Distribution of the Disease. Rev. Soc. Bras. Med. Trop. Uberaba, v. 47, n. 1, jan-fev. 2014. Disponível em: $<$ http://www.scielo.br/scielo.php?script=sci_arttext\&pid=S0037-86822014000100063〉. Acesso em: 01 out. 2018. 